Boletín de la Sociedad Geológica Mexicana

VOLUMEN 64, NÚM. 1, 2012, P. 79-89

\title{
Adobes en arquitectura monumental: análisis químico-físicos, arqueología y reconstrucción 3D para determinar las técnicas constructivas en los sitios de La Joya (México) y Arslantepe (Turquía)
}

\author{
Giovanna Liberotti ${ }^{1, *}$, Annick Daneels ${ }^{2}$ \\ ${ }^{1}$ Universidad de L'Aquila, Facultad de Ingeniería Química y Materiales, Monteluco di Roio, 67100 L'Aquila, Italia. \\ ${ }^{2}$ Instituto de Investigaciones Antropológicas, Universidad Nacional Autónoma de México UNAM, 0451 México D.F. \\ *giovanna.liberotti@gmail.com
}

\section{Resumen}

Este trabajo trata de profundizar en los conocimientos sobre las materias primas usadas en el proceso de elaboración de arquitectura monumental de tierra en sitios arqueológicos. De hecho, a pesar de que la tierra como material de construcción goza de una amplia difusión en el mundo, hay poca información sobre las modalidades bajo las cuales la materia prima ha sido transformada en elementos constructivos y arquitectónicos en la antigüedad. Por lo tanto, se propone una investigación de tipo "arquitectónico", comparando algunos de los edificios de dos sitios pluriestratificados: Arslantepe (V milenio aC - VII s. dC), en la Provincia de Malatya, Turquía, y La Joya, un sitio del período Clásico (I milenio dC), en el Estado de Veracruz, México.

La metodología utilizada para lograr los objetivos está constituida por: a) el análisis químico y físico de los materiales de construcción (muestras de adobes, rellenos y recubrimientos); b) el análisis arquitectónico de los aparejos y de los sistemas constructivos; y c) la reconstrucción virtual 3D de los edificios principales, sustentada en la evidencia arqueológica. El uso combinado de estas herramientas tan diferentes permitió explicar y contextualizar con la atención debida unos rasgos importantes de la arquitectura de tierra de estas dos culturas.

De los resultados preliminares, se sugiere que los antiguos constructores tenían pleno conocimiento del comportamiento de los materiales de construcción en un entorno determinado, adoptando las soluciones más eficaces. El conjunto de todos los datos analizados hasta ahora demuestra en ambas localidades el desarrollo de un diseño arquitectónico muy sofisticado. Los cálculos estructurales para la distribución de la carga, la elección de las tierras más apropiadas para cada función, la "tecnología de los agregados" en la preparación de la pasta y otros temas relevantes desarrollados en el curso de este trabajo prueban que los mecanismos que regulan la arquitectura de tierra, derivados empíricamente a lo largo de años de práctica, son más eficazes de lo que se pensaba hasta ahora.

Palabras clave: Arquitectura de tierra, materiales, arqueología, reconstrucción 3D.

\section{Abstract}

This paper aims to further the knowledge of the raw materials used in the elaboration processes of the earthen architecture in archaeological sites. Although earth as a building material has a wide distribution in the world, there is little information on the way raw materials were processed in antiquity. For this reason, we are proposing an "architectural" study comparing some of the buildings of two pluristratified sites: on the one hand Arslantepe (V millennium BC-VII century AD), in the Province of Malatya, Turkey, and on the other hand La Joya (I millennium AD), in the State of Veracruz, Mexico.

The methodology used to achieve the objectives is based on: a) the chemical, physical and mechanical analysis of building material samples-mud-bricks, mud-plasters and fills; b) the architectural analysis of wall bonding and construction systems; and c) the 3D virtual reconstruction of the major buildings, supported by archaeological evidence. The combination of these different lines of evidence 
allowed explaining and contextualizing the main features of earthen architecture in these two cultures.

Preliminary results reveal that the ancient builders developed a profound knowledge of the possibilities of the building materials in a given environment, empirically deriving the most effective solutions. All the data analyzed so far show a highly sophisticated architectural design on both sites. The structural calculations for load distribution, the choice of the most appropriate sediments for each architectural function, "the aggregate technology" in the mud-brick and mud-plaster mixtures, and other relevant topics developed in this work prove that mechanisms regulating the earthen architecture, when transmitted overtime, are actually more effective than previously thought.

Keywords: Earthen architecture, materials, archaeology, 3D reconstruction.

\section{Introducción}

Las construcciones en tierra fueron entre las primeras soluciones de abrigo artificial que el hombre llevó a cabo, a partir del momento en que desarrolló su actividad de forma sedentaria. Aunque el paso de los siglos ha cubierto muchos de estos sitios tempranos de ocupación humana, no se ha perdido el hábil uso de este material, que surge de conocimientos que se fueron refinando durante el transcurso del tiempo, desde la construcción de chozas y viviendas modestas, hasta palacios, conjuntos urbanos y ciudades enteras (Galdieri, 1982; Achenza y Sanna, 2006). Según Grandreau y Delboy (2010), el 17\% de los lugares en su lista "Patrimonio de la Humanidad" son sitios de arquitectura de tierra y alrededor del $30 \%$ de la población mundial vive en casas hechas de este material. Sin embargo, su importancia no está bien conocida (Alva-Balderrama, 2003). En 1972 se organizó en Yazd, Irán, la primera conferencia internacional sobre la conservación del patrimonio arquitectónico de tierra. A partir de entonces, se activó la investigación en el tema (Avrami et al., 2008), pero no existe todavia un estudio sistemático de las tecnologías constructivas en tierra y de los procesos de elaboración de la materia prima.

Entre los sectores de la construccion que promueven la arquitectura de tierra como alternativa económica y sostenible para la vivienda, ya que es un material totalmente reciclable que requiere de muy poca energía para ser elaborado. Sin embargo, los procedimientos para la caracterización de las muestras en la arquitectura moderna no son directamente aplicables al estudio de monumentos arqueológicos. Asimismo, a pesar de la evolución reciente y actual de la tecnología digital en el manejo de los datos arqueológicos, la aplicación de instrumentos digitales modernos al estudio de la arquitectura de tierra, y sobretodo las consecuencias que derivan de la misma aplicación, están insuficientemente mencionadas en la literatura (Matero et al., 2000; Amici, 2008).

Este trabajo trata de contribuir al conocimiento de los procesos de producción a través de los cuales los antiguos constructores transformaban la materia prima (la tierra), en elementos arquitectónicos, viendo de cerca la técnica de construcción utilizada en dos casos diferentes en los sitios arqueológicos de (1) Arslantepe, en la Provincia de Malatya, Turquía, y (2) La Joya, en el Estado de Veracruz, México. Para lograr el objetivo se utilizarán tres canales de investigación: (a) el análisis químico y físico de muestras de adobes, rellenos y recubrimientos, (b) el análisis arquitectónico de las mamposterías, y (c) la reconstrucción 3D de los edificios principales.

La cantidad de datos recogidos en Arslantepe es considerable, por ser muy larga la duración del proyecto (50 años en el 2011). Por ese motivo, muchos de los análisis programados (sedimentología, láminas delgadas, sales solubles, etc.), considerados estándares en la literatura especializada (Avrami et al., 2008), no han sido realizados todavía. Sin embargo, la investigación hasta ahora ya aporta una contribución importante para entender los conocimientos ingenieriles tanto de las poblaciones anatólicas como de las mesoamericanas.

\section{2. Áreas de estudio}

\subsection{Arslantepe}

El sitio arqueológico de Arslantepe se encuentra en la llanura de Malatya, en Anatolia oriental, un oasis dentro de las montañas del Anti-Taurus, $15 \mathrm{~km}$ al sur del río Éufrates (Figura 1a). Sus coordenadas geográficas son 38²2'54.84" $\mathrm{N}$ y $38^{\circ} 21^{\prime} 38.96^{\prime \prime} \mathrm{E}$, siendo su altitud promedio de 921 $\mathrm{m}$, con un clima templado semiárido, con precipitaciones entre 400 y $600 \mathrm{~mm}$ al año. Es un tell, palabra árabe que define un montículo artificial formado por los depósitos acumulados de muchos asentamientos construidos en el mismo lugar (Frangipane, 2000). Sus dimensiones son 30 $\mathrm{m}$ de altura y $40000 \mathrm{~m}^{2}$ de superficie. Ha sido ocupado sin interrupción desde el V milenio a.C. hasta el periodo romano y bizantino (400-600 d.C.). Desde décadas se vienen realizando campañas de excavación de la Misión Arqueológica Italiana en la Anatolia Oriental (MAIAO, bajo la dirección de M. Frangipane).

Con base en los estudios geológicos e hidromorfológicos 


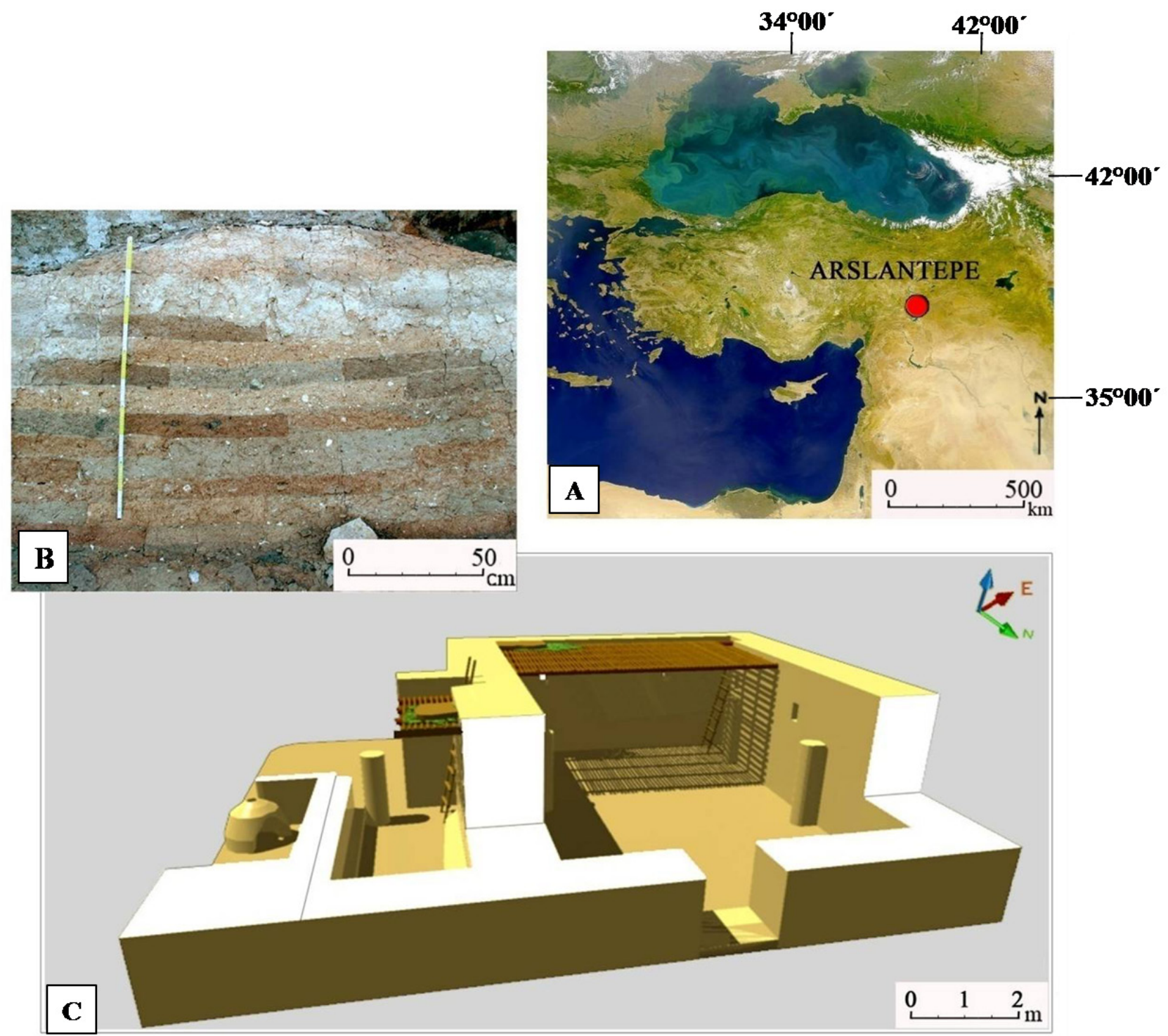

Figura 1. Arslantepe: A) Localización del área de estudio: fuente GoogleMap; B) imagen de uno del los aparejos de período Calcolítico Tardío: tomada por Giovanna Liberotti, Agosto 2008; C) reconstrucción virtual 3D del edificio A582 - vista desde el noreste: autor Giovanna Liberotti.

del territorio (Palmieri, 1978; Palmieri y Marcolongo, 1983), fue posible documentar que las zonas montañosas en el norte y suroeste de la llanura de Malatya están constituidas por mármol, basalto. gneises y esquistos, mostrando erosión. Arslantepe se ubica sobre suelos lacustres formados por arcillas calcáreas, capas de arena y caliza. Hacia el noreste del sitio emerge la forma sub-elíptica del Gelincik Tepe, una masa de roca eruptiva constituida por andesitas, traquitas y espilitas. La zona oriental de la llanura de Malatya se caracteriza por la presencia de una capa freática que explica el alto contenido de humedad de los suelos y la aparición de numerosos manantiales locales. Estas características hidrogeológicas crean condiciones favorables para el riego por gravedad durante los períodos secos, por lo que el área es una región agrícola ideal. Los estudios de paleobotánica
(Sadori et al., 2008) han demostrado, para el periodo indagado (Calcolítico Tardío, 3850-3000 a.C.) la presencia de bosques de clima templado, ahora extintos, de pino, fresno, enebro y álamo.

\subsection{La Joya}

La Joya es una ciudad prehispánica con edificios de tierra, ubicada en la confluencia de los ríos Jamapa y Cotaxtla, en el municipio de Medellín de Bravo, Estado de Veracruz, México (Figura 2a). Sus coordenadas geográficas son $19^{\circ} 04^{\prime} 00^{\prime \prime} \mathrm{N} 96^{\circ} 09^{\prime} 00^{\prime \prime} \mathrm{W}$, siendo su altitud de $7 \mathrm{~m}$. Ambos ríos tienen su origen en las faldas del volcán Pico de Orizaba, y cruzan una estrecha franja de calizas en su camino a la costa, proporcionando abundantes arcillas del 


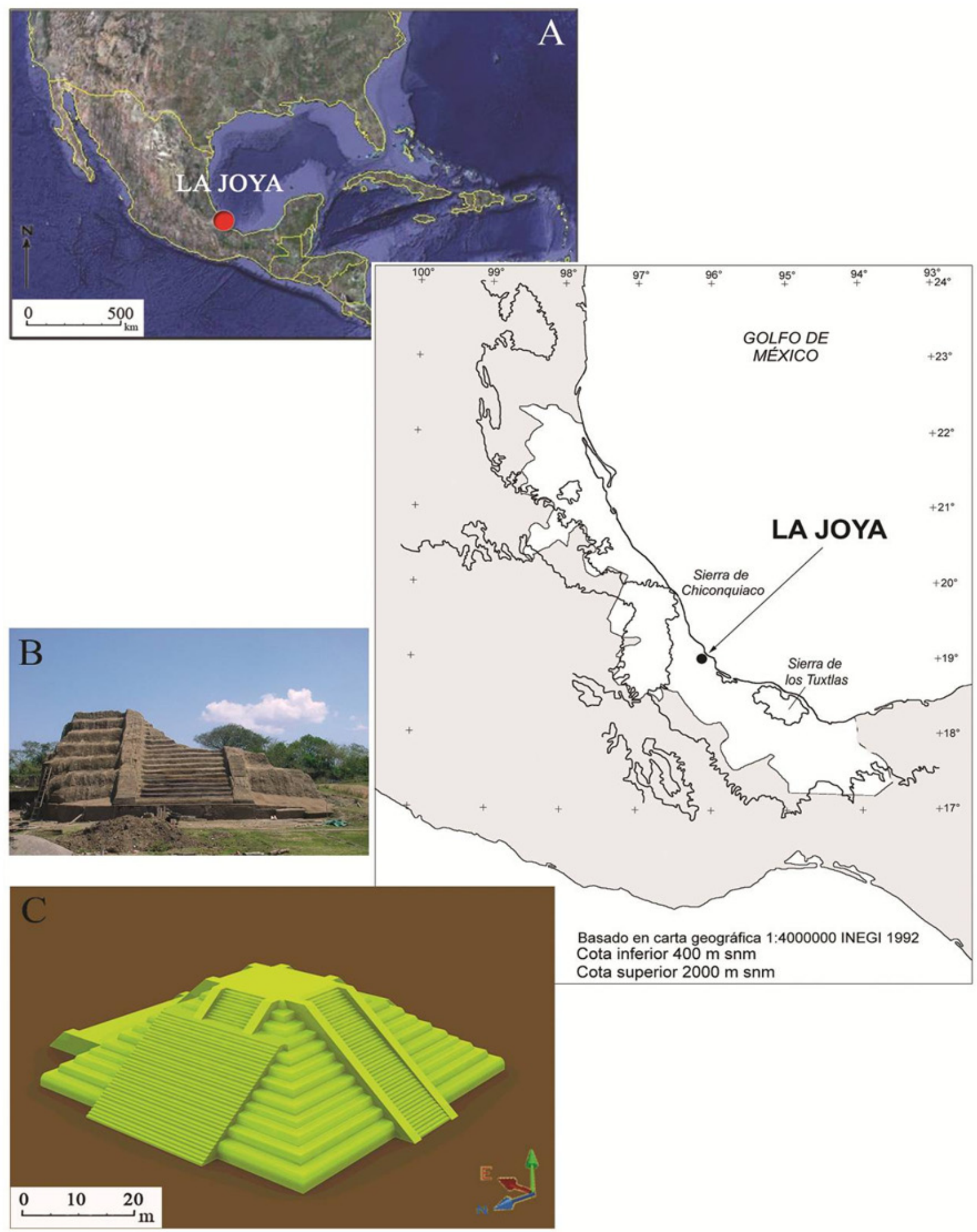

Figura 2. La Joya: A) localización del área de estudio: fuente GoogleMap e INEGI (1992); B) imagen de la pirámide: tomada por la Dra. Annick Daneels, Febrero 2009; C) reconstrucción virtual 3D de la pirámide: autor Giovanna Liberotti. 
tipo expansivo a lo largo de las terrazas aluviales. El área de tierras bajas tiene una precipitación media anual de alrededor de $1500 \mathrm{~mm}$, producida sobre todo entre mayo y noviembre (es dos veces mayor en las tierras bajas más al sur, donde están los sitios olmecas). Durante el invierno, vientos huracanados recorren las llanuras costeras. En estas condiciones geomorfológicas y climáticas, la arquitectura de tierra no parecería ser una opción muy prometedora. Sin embargo, la investigación comprueba su viabilidad en un ambiente de trópico húmedo, en condiciones de alta precipitación, proporcionando una importante contribución a la comprensión de la cultura arqueológica regional (cultura de Remojadas o del Centro-Sur de Veracruz).

La secuencia del sitio abarca el primer milenio de nuestra era. Hasta la fecha se han logrado exponer algunas partes conservadas de los edificios mayores, completamente construidos de tierra (la pirámide principal y dos palacios) que delimitan la plaza principal. Se ha podido observar el rápido deterioro de las superficies expuestas durante el proceso de excavación arqueológica. Este hecho contrasta con la buena conservación de los acabados antiguos, cada uno de los cuales se ha comprobado que duró decenas, hasta cientos de años en uso, con sólo remozamientos ocasionales (Daneels, 2008b).

\section{Metodología}

La propuesta metodológica que constituye la base de esta investigación consiste en combinar diversos instrumentos: (a) el análisis químico, físico y mecánico de la tierra utilizada en la construcción de los edificios antiguos, para verificar el comportamiento del material, (b) el análisis de los tipos de sistemas constructivos, buscando las razones de su transformación/continuidad, para comprender el contexto cultural y el conocimiento técnico, y (c) la reconstrucción virtual 3D de los edificios principales, para aclarar algunos aspectos esenciales de los sistemas de construcción utilizados.

En Arslantepe se muestrearon dieciséis adobes de período Calcolítico Tardío, elegidos en base a la diferencia de color, y siete muestras de aplanado, seleccionados con base en su estado de conservación. La caracterización de las muestras se efectuó mediante:

1. Difracción por rayos X, con un Difractómetro Philips Analitycal X'Pert; en todas las muestras se separó la fracción $<2 \mu \mathrm{m}$ y se prepararon y analizaron los agregados orientados siguiendo los lineamientos propuestos por Giampaolo y Lo Mastro (2000);

2. Fluorescencia de rayos $\mathrm{X}$, con un espectrómetro modelo Spectra 2000;

3. Microscopía electrónica de barrido con un aparato SEM Philips XL30, en gránulos seleccionados de las muestras 1, 4, 7, 9, 13, 16 y en fragmentos de fibras fósiles recogidas de la muestra 12 , puestos en adhesivos y recubiertos con una capa delgada de oro;

4. Densometría, para determinar densidad real y aparente, cuantificadas mediante un picnómetro de helio modelo AccuPyc 1330;

5. Porosimetría, mediante un micrómetro modelo GeoPyc 1360; para cada muestra se seleccionó un gránulo de ca. $1 \mathrm{~cm}$ que fue colocado en una base de Dryflo (óxido de aluminio y grafito) y ubicado en el tubo de medida.

En La Joya han sido analizadas hasta la fecha 20 muestras procedentes de la Pirámide y de los edificios en la Plataforma Este y Norte. Se seleccionó una muestra de los distintos materiales constructivos (adobes, pisos, recubrimientos, rellenos), de distintos edificios y etapas constructivas, para evaluar de manera inicial el rango de variación y cambios de orden cronológico. Para caracterizar formalmente a las muestras se efectuaron:

1. Difracción de rayos $\mathrm{X}$, con un difractómetro Philips modelo 1130/96 (generador) y PW1050/25 (goniómetro), utilizando radiación $\mathrm{CuK} \alpha$. $\mathrm{La}$ fracción de arcilla fue tratada con base al protocolo de Girón (2010). De cada muestra se analizó fracción orientada saturada con $\mathrm{MgCl}_{2}$, fracción orientada glicolada y fracción orientada calcinada a $550{ }^{\circ} \mathrm{C}$;

2. Fluorescencia de rayos $\mathrm{X}$, con la muestra en base seca, usando el espectrómetro Siemens SRS3000. La pérdida por calcinación (PXC) se determinó calcinando $1 \mathrm{~g}$ de muestra a $950^{\circ} \mathrm{C}$ durante 1 hora;

3. Análisis petrográfico de láminas delgadas, usando microscopio binocular con luz natural y luz polarizada, para la descripción de fábrica, textura, mineralogía cualitativa y clasificación geológica;

4. Sedimentación y límites de Atterberg con base a la metodología de Reddy (2002);

5. Espectroscopía FT-IR (espectroscopia de infrarrojo por transformada de Fourier) sobre muestras de revestimientos, para encontrar un aglutinante orgánico que explique la resistencia de las fachadas antiguas a condiciones climáticas desfavorables, considerando el óptimo estado de conservación de las superficies tras ser excavadas. Se utilizó el Espectrofotómetro de Infrarrojo FT-IR marca Thermo Scientific, modelo Nicolet 6700. La toma de los espectros se realizó en el modo ATR (reflectancia total atenuada) con un accesorio marca Thermo Scientific, modelo Smart Orbit. Se utilizaron los siguientes parámetros: número de barridos, 64, número de barridos del fondo analítico, 64, resolución de 4000, ganancia de 4. Se determinó el fondo analítico y posteriormente el espectro de IR de la muestra en forma de polvo.

El análisis arquitectónico de las estructuras principales se llevó a cabo midiendo el tamaño de los adobes pertenecientes a las mamposterías estudiadas y observando cuidadosamente 
todos los elementos aplicados (cimentaciones, morteros, aplanados, etc.). En Arslantepe, se dibujaron las elevaciones frontales de los muros donde se colectaron las muestras, para mostrar las fachadas en detalle. En La Joya, se estudiaron los componentes estructurales de los edificios más grandes, la textura, el método de colocación de los adobes, y el sistema de techado de unos edificios.

Las reconstrucciones virtuales $3 \mathrm{D}$, basadas en las evidencias arqueológicas registradas por la persona que excavó los edificios, se llevaron a cabo a partir de los dibujos (planimetrías y secciones estratigráficas) hechos directamente en el sitio de Arslantepe. Para La Joya se recolectaron los dibujos originales hechos por los arqueólogos en las campañas anteriores. Cada modelo tridimensional ha sido creado con el software AutoCAD 2008 y elaborado en Photoshop.

\section{Contexto de procedencia de los materiales estudiados}

\subsection{Arslantepe}

Los cuatro aparejos de período Calcolítico Tardío de donde se recogieron dieciseis muestras de adobe pertenecen a cuatro edificios nombrados A582, A853, A842, A950, atribuibles a etapas constructivas consecutivas (Frangipane, 2002). El primero es una amplia habitación de $70 \mathrm{~m}^{2}$, con muros perimetrales de $1 \times 1,20 \mathrm{~m}$ de espesor. Las pinturas en las paredes y la presencia de columnas sugieren que tuvo una función importante (vivienda de familias de alto rango). El edificio A853 es una pequeña habitación, con muros poco espesos. La habitación A842 es larga y estrecha ( $2 \times 10 \mathrm{~m})$ y pertenece, junto con otras habitaciones similares, a un área de almacenamiento. La habitación A950 es parte de un edificio ceremonial de 20x22 m construido sobre una plataforma de losas de piedra y postes de madera. Las siete muestras de aplanado analizadas pertenecen a unas estructuras del conjunto palaciego (Alvaro, 2004): A364, A365, A796, A135, A450 y A830. Estos edificios monumentales de adobe se encuentran en el lado oeste del tell, y poseen gran importancia ya que demuestran el papel clave desempeñado por Arslantepe en el proceso de formación del estado en Anatolia (Frangipane, 2000).

\subsection{La Joya}

En La Joya han sido analizadas:

- 5 muestras de adobe de un recinto de la tercera etapa constructiva de la Plataforma Este,

- 4 de pisos exteriores (no protegidos por techo) de la segunda y de la tercera etapa constructiva de las Plataformas Este y Norte,

- 2 fragmentos de un recubrimiento del muro exterior de un recinto de la segunda etapa constructiva de la Platafoma Norte,
- 1 del firme de piso de plaza al pie de la Pirámide (adosamiento a la primera etapa constructiva),

- 7 de rellenos de las distintas texturas encontradas, desde arcilla a arena, de la primera a cuarta etapa constructivas de los distintos edificios, y

- como control, 1 muestra de arena gris moderna, usada para la producción contemporánea de ladrillos, extraída localmente de dunas costeras y usada para la producción contemporánea de ladrillos (Daneels, 2008a).

La estructura más relevante de todo el sitio es la Pirámide, de la cual fue posible identificar sólo los restos de la fachada oeste con su escalera central de $6 \mathrm{~m}$ de ancho, con alfardas laterales (Figura 2b). Gracias a las excavaciones ha sido posible reconstruir el tamaño y la apariencia de la estructura original, con una superficie de $45 \mathrm{~m}$ de lado y escalinatas en los cuatro lados.

\section{Resultados}

\subsection{Arslantepe}

El análisis por difracción por rayos $\mathrm{X}$ demuestra que las matrices de las muestras comparten la misma composición mineralógica (calcita y cuarzo), variando en las fases subordinadas (plagioclasas, ankerita, yeso, dolomita). Esto indica que el origen de las materias primas utilizadas para la fabricación de los adobes es local: proceden de suelos calcáreos que contienen arenas cuarzosas, minerales secundarios y fracción arcillosa. En esta última, la ilita y la caolinita son las fases predominantes, seguidas de clorita y, más escasa, la montmorillonita. Este último componente se caracteriza por secarse con dificultad y una alta capacidad de contracción, por lo que los adobes con el menor contenido posible en montmorillonita son los que permiten una mayor eficiencia en el proceso de construcción. La diferente plasticidad de las muestras se explica por el distinto contenido porcentual de fases arcillosas, de manera que los adobes que contienen caolinita en mayor cantidad son más plásticos. Los resultados de los análisis de fluorescencia por rayos X se presentan en la Tabla 1, e indican que la composición química de los materiales es similar. Las muestras que contienen menor cantidad de calcio $(8,10$, $13,14,15,16)$ y mayor cantidad de silicio y aluminio, son más ricas en fracción arcillosa. Este es el caso de la serie de muestras de adobes de 13 a 16, que pertenecen a una de las habitaciones del edificio ceremonial (A950). Sin embargo, el calcio es un consolidante natural en la tierra cruda (Guerrero-Baca y Uviña, 2009), lo que significa una ventaja en la materia prima usada para la construcción en Arslantepe. La porosidad es visible al microscopio óptico y mediante microscopía electrónica de barrido. Los resultados indican una correlación entre porosidad y el uso de fibras vegetales como complemento en la mezcla de tierra y agua (Liberotti y Quaresima, 2010). Con respecto a los 
Tabla 1. Características químicas y físicas de los materiales de Arslantepe*

\begin{tabular}{|c|c|c|c|c|c|c|c|c|c|c|c|}
\hline $\begin{array}{c}\mathrm{N}^{\circ} \\
\text { muestra }\end{array}$ & Edificio & $\begin{array}{l}\mathrm{Al} \\
(\%) \\
\end{array}$ & $\begin{array}{l}\mathrm{Mg} \\
(\%) \\
\end{array}$ & $\begin{array}{c}\mathrm{Fe} \\
(\%) \\
\end{array}$ & $\begin{array}{r}\mathrm{Si} \\
(\%) \\
\end{array}$ & $\begin{array}{r}\mathrm{Cl} \\
(\%) \\
\end{array}$ & $\begin{array}{c}K \\
(\%) \\
\end{array}$ & $\begin{array}{l}\mathrm{Ca} \\
(\%) \\
\end{array}$ & $\begin{array}{c}\text { Porosidad } \\
(\%) \\
\end{array}$ & $\begin{array}{c}\text { Densidad } \\
\text { Real } \\
\left(\mathrm{g} / \mathrm{cm}^{3)}\right. \\
\end{array}$ & $\begin{array}{c}\text { Densidad } \\
\text { Aparente } \\
\left(\mathrm{g} / \mathrm{cm}^{3)}\right.\end{array}$ \\
\hline 1 ad. & A582 & 5.88 & 4.88 & 7.19 & 29.41 & 3.85 & 6.42 & 40.31 & 27.95 & 2.618 & 1.912 \\
\hline 2 ad. & A582 & 3.99 & 4.57 & 4.51 & 17.76 & 9.01 & 5.13 & 53.73 & 38.33 & 2.651 & 1.635 \\
\hline 3 ad. & A582 & 5.81 & 7.21 & 5.36 & 28.33 & 3.11 & 7.01 & 41.89 & 41.47 & 2.633 & 1.552 \\
\hline 4 ad. & A582 & 6.19 & 7.74 & 5.91 & 29.63 & 2.18 & 6.46 & 40.06 & 29.03 & 2.652 & 1.882 \\
\hline 5 ad. & A582 & 4.55 & 10.96 & 5.86 & 25.21 & 2.31 & 5.44 & 43.8 & 36.44 & 2.651 & 1.685 \\
\hline 6 ad. & A582 & 4.09 & 6.01 & 5.01 & 19.54 & 12.14 & 6.21 & 45.93 & 40.38 & 2.655 & 1.581 \\
\hline 7 ad. & A582 & 5.25 & 9.18 & 6.56 & 27.73 & 2.37 & 5.87 & 41.25 & 28.63 & 2.654 & 1.883 \\
\hline 8 ad. & A582 & 7.28 & 6.47 & 8.76 & 31.48 & 2.53 & 7.23 & 34.45 & 22.01 & 2.665 & 2.079 \\
\hline 9 ad. & A853 & 6.01 & 5.33 & 6.19 & 25.43 & 1.42 & 4.46 & 49.48 & - & - & - \\
\hline $10 \mathrm{ad}$. & A853 & 9.75 & 5.42 & 8.57 & 37.98 & 0.93 & 5.84 & 29.84 & 36.64 & 2.831 & 1.681 \\
\hline $11 \mathrm{ad}$. & A 842 & 6.95 & 6.08 & 7.06 & 29.84 & 4.11 & 6.43 & 37.74 & 38.46 & 2.651 & 1.631 \\
\hline $12 \mathrm{ad}$. & A 842 & 6.85 & 6.54 & 6.64 & 29.02 & 1.16 & 4.68 & 43.76 & 38.93 & 2.714 & 1.657 \\
\hline $13 \mathrm{ad}$. & A950 & 9.93 & 5.51 & 7.71 & 37.79 & 0.76 & 6.06 & 30.71 & - & - & - \\
\hline $14 \mathrm{ad}$. & A950 & 8.75 & 5.35 & 8.48 & 34.85 & 5.09 & 6.82 & 28.55 & 23.21 & 2.651 & 2.021 \\
\hline $15 \mathrm{ad}$ & A950 & 8.34 & 5.24 & 8.06 & 35.83 & 0.81 & 5.67 & 34.33 & - & - & - \\
\hline $16 \mathrm{ad}$. & A950 & 11.1 & 4.75 & 11.35 & 41.12 & 2.82 & 7.04 & 19.37 & 23.51 & 2.713 & 2.077 \\
\hline 17 apl. & A365 & 7.48 & 6.21 & 6.89 & 36.76 & 0.04 & 3.31 & 37.55 & 57.73 & 2.681 & 1.132 \\
\hline 18 apl. & A364 & 3.74 & 5.58 & 6.91 & 28.71 & 6.84 & 7.18 & 51.81 & 53.26 & 2.582 & 1.213 \\
\hline 19 apl. & A796 & 6.23 & 6.53 & 6.02 & 30.98 & 3.91 & 5.14 & 39.49 & 45.92 & 2.561 & 1.381 \\
\hline 20 apl. & A135 & 5.97 & 6.22 & 5.78 & 31.37 & 2.51 & 7.45 & 38.63 & 51.52 & 2.683 & 1.315 \\
\hline 21 apl. & A830 & 5.62 & 6.29 & 5.74 & 28.67 & 3.65 & 5.83 & 41.88 & 50.45 & 2.591 & 1.281 \\
\hline 22 apl. & A 830 & 5.74 & 6.51 & 5.88 & 29.83 & 4.47 & 5.17 & 40.32 & 40.61 & 2.674 & 1.513 \\
\hline 23 apl. & $\mathrm{A} 450$ & 5.55 & 4.24 & 6.82 & 31.21 & 0.62 & 3.93 & 45.66 & 56.25 & 2.725 & 1.191 \\
\hline
\end{tabular}

* Los resultados se expresan como porcentaje de óxidos normalizada a 100, para facilitar la comparación. ad. = adobe; apl. = aplanado.

porcentajes de porosidad detectados, que se presentan en la Tabla 1, parece posible dividir las muestras en cuatro clases diferentes. La primera consiste en un valor bajo $( \pm 22 \%)$, lo que significa una baja proporción de material agregado en la elaboración de los adobes. La segunda clase se caracteriza por un valor medio $( \pm 30 \%)$, mientras que la tercera es marcada por una alta porosidad ( $\pm 40 \%)$, indicando una alta cantidad de fibra en la masa. Finalmente, en la cuarta clase, que incluye las muestras de aplanado (17-23), la porosidad es bastante mayor, alcanzando el $58 \%$. La macroporosidad puede justificar la buena condición actual de los adobes y los aplanados, que tienen alta permeabilidad al vapor y un secado muy rápido. Los resultados que se presentan en la Tabla 1 muestran que en la densidad real y aparente de todas las muestras analizadas no hay cambios significativos. Sin embargo se observó que la densidad real media obtenida para Arslantepe $\left(2.68 \mathrm{~g} / \mathrm{cm}^{3}\right)$, supera la media entre 1.6 y $2.2 \mathrm{~g} / \mathrm{cm}^{3}$ reportada en otros trabajos (e.g., Achenza y Sanna, 2006).

El análisis arquitectónico de las evidencias de periodo Calcolítico Tardío demuestra que los adobes que constituyen los edificios más antiguos (A582 y A853; Figura 1b) son muy irregulares en la forma (de 6x20x25 a 8x30x90 cm), mientras que los adobes de los edificios cronológicamente posteriores son más regulares (de $7 \times 30 \times 60$ a $8 \times 35 \times 65$ $\mathrm{cm})$. Este aspecto está ilustrado por la aplicación, en las paredes de los edificios tempranos A582 y A853, de una mayor cantidad de mortero entre las hileras de adobes, a fin de compensar las irregularidades de las superficies de apoyo. Con respecto a la cimentación, la parte inferior de las paredes casi siempre consta de tierra compacta, homogénea y rica en materia inerte: esta técnica puede realizar la doble función de atar las piedras y proporcionar una base para la elevación del muro.

En la reconstrucción virtual 3D del edificio A582 se destaca la magnitud de la sala rectangular y la complejidad del sistema de techado (vigas de madera, cañas entrelazadas y barro), que fue reconstruido de acuerdo a los fragmentos hallados en el derrumbe sobre los pisos. En la Figura 1c se muestra la estructura de un piso, aunque el espesor de las paredes puede asumir dos o más pisos. Se supone que la entrada al edificio estaba situada en la pared norte, donde las excavaciones han revelado una superficie de aplanado con una ligera pendiente hacia el norte.

\subsection{La Joya}

Los resultados obtenidos hasta la fecha de los análisis de las muestras en La Joya (Daneels y Guerrero-Baca, 2011) muestran un material de construcción muy coherente mineralógicamente. Los minerales principales son feldespatos, plagioclasas y cuarzo, y se presentan bandas que indican la presencia de arcillas. Hay poco material amorfo, tal vez vítreo. En las fracciones orientadas se identifica 
clorita y esmectita (montmorillonita). Existen indicios de caolinita, pero su proporción debe ser muy baja por los tipos de depósitos geológicos, principalmente volcánicos recientes, de donde proceden las arcillas en la región. Ello se confirma por medio de estudios petrográficos, mediante los que se determinó un origen andesítico-dacítico para la matriz y los minerales asociados. La fracción fina está, por lo tanto, compuesta principalmente de arcillas muy expansivas, de difícil manejo para la construcción. Los adobes están conformados por sedimentos con contenidos de arena, limo y arcilla variables, mezclados con paja picada. A diferencia de Arslantepe, los fragmentos de cerámica, huesos y carbón (si bien los hay ocasionalmente como se observó en algunas de las láminas delgadas de La Joya; Figura 3a), sólo están presentes como inclusiones accidentales. Por su parte, los revestimientos, de 0.013 a $0.018 \mathrm{~mm}$ de espesor, constan de una mezcla con una alta proporción de arcilla (60-70 $\%$ de fracción fina) y paja finamente picada (Figura 3b), identificada como género Panicum en una proporción de hasta 30 a $40 \%$, de acuerdo a las tablas de estimación de Folk (1951). El estudio petrográfico de las capas de recubrimiento indica una mezcla de suelos procedente de diferentes perfiles (Figura 3c-d). Eso podría sugerir una extracción de la materia prima en diferentes lugares. Las muestras parecen también indicar que hubo una selección cuidadosa de la fracción granulométrica en la preparación de los aplanados, con una matriz arcillosa (gleyica) combinada con un aporte de granos angulares bien graduados en las capas de firme, y una fracción mucho más fina para las delgadas capas de aplanado de superficie. Sin embargo, para desarrollar esta temática y aclarar la relación entre función arquitectónica y granulometría del material usado se necesitan análisis de láminas delgadas trasversales. El análisis FTIR sobre muestras de revestimientos sugiere la presencia de un componente orgánico parecido al mucílago de nopal, cuyo uso está atestiguado en las tierras altas de México prehispánico hasta la época moderna (GuerreroBaca, 2007). Los resultados de los límites de Atterberg que se presentan en la Tabla 2, se encuentran dentro del promedio. Los resultados del análisis de fluorescencia por rayos X (Tabla 2) indican que el origen del material utilizado es local. Las variaciones pueden derivar de una
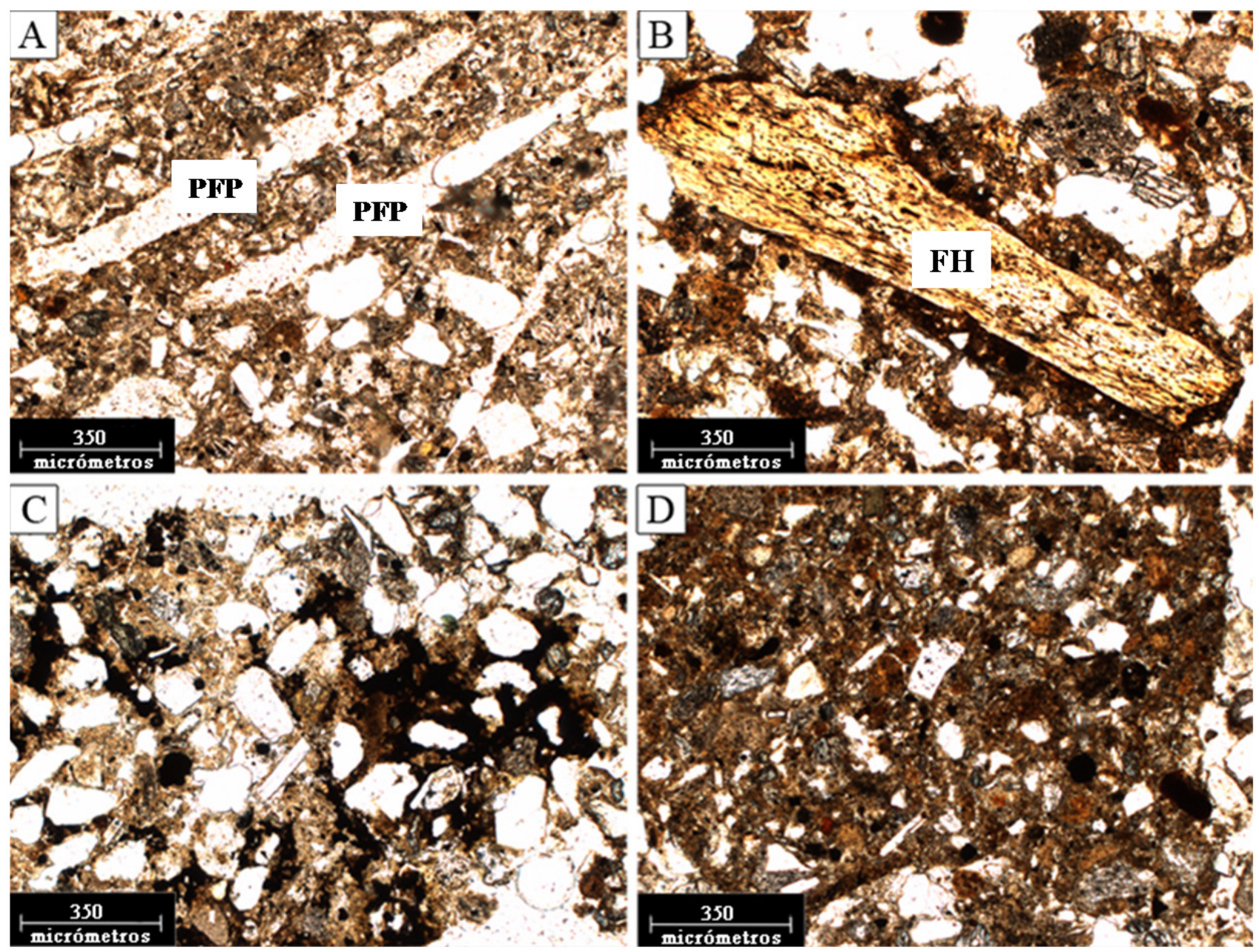

Figura 3. Micromorfología del suelo en La Joya: A) PFP = Poros de Fragmentos de Paja: poros alargados discontinuos debidos a los fragmentos de paja utilizada en la pasta, B) fragmento de hueso FH, C) suelo gléyco en muestra de revestimiento, D) suelo rojo en muestra de revestimiento. 
Tabla 2. Características químicas y físicas de los materiales de La Joya*.

\begin{tabular}{|c|c|c|c|c|c|c|c|c|c|c|c|}
\hline $\begin{array}{c}\mathrm{N}^{\circ} \\
\text { muestra }\end{array}$ & Edificio & $\begin{array}{l}\text { Al } \\
(\%) \\
\end{array}$ & $\begin{array}{l}\mathrm{Mg} \\
(\%) \\
\end{array}$ & $\begin{array}{l}\mathrm{Fe} \\
(\%) \\
\end{array}$ & $\begin{array}{c}\text { Si } \\
(\%) \\
\end{array}$ & $\begin{array}{c}K \\
(\%) \\
\end{array}$ & $\begin{array}{l}\mathrm{Ca} \\
(\%) \\
\end{array}$ & $\begin{array}{c}\text { Límite } \\
\text { Líquido } \\
(\%)\end{array}$ & $\begin{array}{c}\text { Límite } \\
\text { Plástico } \\
(\%) \\
\end{array}$ & $\begin{array}{c}\text { Indice de } \\
\text { Plasticidad } \\
(\%) \\
\end{array}$ & $\begin{array}{l}\text { Clasificación } \\
\text { SUCS** }\end{array}$ \\
\hline 1 ad. & Pl.E. & 17.7 & 1.73 & 7.66 & 60.57 & 1.38 & 3.25 & 28.9 & 19.2 & 9.7 & $\mathrm{SC}$ \\
\hline 2 ad. & Pl.E. & 17.85 & 1.6 & 7.45 & 61.81 & 1.4 & 3.18 & 28.1 & 19.3 & 8.8 & $\mathrm{SC}$ \\
\hline 3 ad. & Pl.E. & 17.52 & 1.6 & 7.63 & 60.44 & 1.38 & 3.22 & 28.6 & 19.2 & 9.4 & $\mathrm{SC}$ \\
\hline 4 ad. & Pl.E. & 17.48 & 1.67 & 7.57 & 60.46 & 1.38 & 3.29 & 31.2 & 20.6 & 10.6 & $\mathrm{SC}$ \\
\hline $5 \mathrm{ad}$ & Pl.E. & 17.6 & 1.58 & 7.55 & 60.51 & 1.39 & 3.18 & 27.5 & 19.6 & 7.9 & $\mathrm{SC}$ \\
\hline 6 apl. & Pl.E. & 16.8 & 1.7 & 6.54 & 63.15 & 1.5 & 3.23 & 25.2 & 18.4 & 6.8 & SM \\
\hline 7 apl. & Pl.N. & 16.04 & 1.98 & 6.92 & 62.03 & 1.43 & 3.95 & 22 & - & - & SM \\
\hline 8 apl. & Pl.N. & 19.45 & 2.01 & 6.88 & 57.43 & 1.23 & 4.17 & 28.4 & 19.6 & 8.8 & $\mathrm{CL}$ \\
\hline 9 apl. & PIR & 19.45 & 1.82 & 7.41 & 55.84 & 1.3 & 3.86 & 28.8 & 19.7 & 9.1 & CL \\
\hline 10 apl. & Pl.E. & 17.96 & 1.44 & 6.55 & 60.42 & 1.57 & 3.44 & 25.7 & 17.8 & 7.9 & $\mathrm{CL}$ \\
\hline 11 apl. & Pl.N. & 15.54 & 1.69 & 6.57 & 64.11 & 1.54 & 3.32 & 24.4 & 6.1 & 6.1 & SM \\
\hline 12 apl. & Pl.N. & 16.05 & 2.37 & 8.97 & 58.44 & 1.34 & 4.08 & 23.9 & - & - & ML \\
\hline 14 rel. & PIR & 14.01 & 1.47 & 5.93 & 65.62 & 1.55 & 3.4 & 23.8 & - & - & SM \\
\hline 15 rel. & PIR & 15.63 & 1.76 & 6.85 & 62.47 & 1.45 & 3.75 & 22 & - & - & $\mathrm{SM}$ \\
\hline 16 rel. & Pl.E. & 21.29 & 0.98 & 7.13 & 55.27 & 1.18 & 1.94 & 39.9 & 22.6 & 17.3 & CL \\
\hline 17 rel. & Pl.E. & 18.29 & 7.43 & 1.7 & 3.51 & 1.29 & 0.14 & 25.9 & 19.3 & 6.6 & SM \\
\hline 18 rel. & Pl.N. & 20.99 & 6.9 & 1.37 & 3.48 & 1 & 0.24 & 34.2 & 21.7 & 12.5 & $\mathrm{CL}$ \\
\hline 19 rel. & Pl.N. & 12.23 & 6.12 & 2.03 & 3.31 & 1.73 & 0.18 & 18.7 & - & - & SM \\
\hline $20 \mathrm{mo}$. & - & 13.76 & 5.03 & 2.55 & 6.7 & 1.86 & 0.14 & - & - & - & - \\
\hline
\end{tabular}

* Los resultados se expresan como porcentaje de óxidos normalizada a 100, para facilitar la comparación.

ad. = adobe; apl. = aplanado; rel. = relleno; mo. = moderna

Pl.E. = Plataforma Este; Pl.N. = Plataforma Norte; PIR. = Pirámide

**Sistema Unificado de Clasificación de Suelos: SC - arena arcillosa; SM - arena limosa; CL - arcilla de baja plasticidad; ML - Limo de baja plasticidad

selección y/o procesamiento de la materia prima (Daneels y Guerrero-Baca, 2011).

El análisis arquitectónico de la Pirámide indica que en la segunda etapa constructiva se aplicó una estrategia original (en el sentido que ésta se reporta por primera vez en el presente trabajo) para controlar la presión interna del relleno de tierra. Las excavaciones muestran que para resolver este problema los constructores alternaron grandes bloques de relleno de arcillas (aproximadamente $1 \mathrm{~m}$ de altura y más de $6 \mathrm{~m}$ de lado) y margas arenosas, ambas disponibles en los alrededores del sitio. Los bloques de arcilla formaron contrafuertes debido a su cohesión. A la inversa, los bloques de suelo arenoso lograron una mejor compactación y por lo tanto una superficie más estable que la arcilla, que tiende a agrietarse en condiciones secas y expandirse en mojado. Para facilitar el transporte del relleno desde el nivel de la plaza, la escalera de la subestructura se mantuvo accesible mediante la construcción de taludes de contención hechos de limo, que se montaron encima de cada una de las alfardas de la escalera, contra los que lindaban los bloques de relleno. Con el llenado del sector de la escalera termina la etapa final del proceso de construcción. En el caso de la primera etapa constructiva de la Pirámide, el relleno es pura arena, probablemente obtenida de la paleoduna, contenida en su perímetro por estratos de limo arcilloso. Con respecto a las Plataformas, tienen viviendas construidas con adobes de tamaño bastante grande: $0.80 \mathrm{~m}$ de largo, $0.10 \mathrm{~m}$ de espesor, $\mathrm{y}$ un ancho que al parecer tiende a decrecer a través del tiempo: de $0.40 \mathrm{~m}$ en la primera etapa constructiva, a $0.35 \mathrm{~m}$ en la segunda, y $0.30 \mathrm{~m}$ a partir de la tercera etapa. La textura de los adobes de los cuartos es bastante similar en todos los niveles y algunas veces, apenas se puede distinguir el mortero de tierra utilizado como cementante. Los adobes se colocaron cuatrapeados longitudinalmente, de manera que cada adobe esté a caballo sobre dos adobes de la hilera de abajo. A pesar de la alta precipitación, la evidencia apunta a tejados planos: en los pisos no hay huellas de horcones de madera, que serían necesarios para apoyar un techo de palma a dos aguas, típico de la arquitectura vernácula tropical. En el derrumbe del techo de una vivienda quemada de la Plataforma Este, se encontraron fragmentos de bajareque cocido en el incendio con la impronta de vigas paralelas estrechas, apenas $1 \mathrm{~cm}$ de diámetro, de corte circular $\mathrm{u}$ oval, atados con cordeles. La ausencia de líneas de goteo en los pisos que rodean las viviendas sugiere una especie de parapeto controlando el escurrimiento pluvial. La boca de una tubería de agua de forma ligeramente cónica en el borde superior de la plataforma basal puede ser indicativa del punto donde la lluvia era drenada del techo.

En la hipótesis de reconstrucción de la Pirámide (Figura 2c), de acuerdo a la inclinación de los escalones de la escalera oeste (la única conservada), se colocaron doce cuerpos, a diferencia de la reconstrucción inicial de nueve cuerpos (Daneels, 2008a). La Pirámide tiene cuatro escaleras, una en cada lado: la oeste es más angosta $(9 \mathrm{~m}$, incluyendo rampas), por ello se puede alcanzar el último 
cuerpo de la pirámide. Por su parte, las escaleras norte y sur tienen un ancho de $20 \mathrm{~m}$, mientras la escalera este era más grande - $25 \mathrm{~m}$, lo que les permite subir no más que a un cierto nivel. Por esta razón fueron reconstruidas virtualmente en dos partes, una inferior y más amplia, y una superior y más estrecha, que llegaba hasta la cumbre.

\section{Discusión y conclusiones}

Como lo muestran los resultados de los análisis, los habitantes anatólicos de Arslantepe, así como los prehispánicos de La Joya, manipularon la materia prima proveniente de su entorno cercano para construir edificios monumentales en tierra cruda. A pesar de las diferencias en tiempo, espacio, cultura, condiciones climáticas y geológicas, hay una convergencia en el uso de adobes con un componente de fibra orgánica para las paredes y techos planos de madera cubiertos de barro. Sin embargo, los estudios realizados muestran claramente cómo se fue desarrollando una tradición propia, con soluciones adaptadas a las condiciones locales.

Los análisis físico-químicos revelaron que la materia prima de Arslantepe es de mejor calidad que la de La Joya, por ser de arcillas poco expansivas (caolinas), naturalmente mezcladas con yeso, cuyo calcio funcionó como un consolidante natural del adobe crudo. Sin embargo, interesante en este sentido es que en la serie de muestras de 13 a 16 de los adobes de A950, el contenido de calcio es inferior a la media, y la proporción de arcilla es la más alta de las muestras. Aunque no sea comprobable, esta característica puede resultar del uso de diferentes tipos de tierra para fabricar la pasta de los adobes, demostrando la búsqueda de una mayor calidad en las materias primas para la construcción de un edificio que podría ser más importante que otros. El análisis de porosidad revela que el $65 \%$ de las muestras analizadas pertenecen a las dos categorías de valores más altos, es decir, de mayor proporción de fibras vegetales en la pasta. No se puede saber con precisión el grado de intencionalidad de la operación, sin embargo se puede tratar de una tecnología específica, cuyo objetivo es controlar la contracción de la arcilla al secar, consolidar mecánicamente la pasta y favorecer una rápida evaporación. En comparación, las muestras de La Joya indican el uso de una arcilla expansiva de tipo smectita (montmorillinita), trabajada igualmente con un componente de zacate picado hasta un porcentaje estimado en un 30 o $40 \%$. Si bien aun carecemos de datos cuantificativos precisos sobre la porosidad de las muestras estructurales de La Joya, parece ser menor que en Arslantepe, en la medida que los estudios aplicados allí relacionan la porosidad con el componente orgánico. Esto puede deberse a la diferencia en el tipo de arcilla, ya que con arcillas expansivas conviene retrasar la evaporación para evitar la formación de grietas.

Los datos obtenidos del análisis arquitectónico pueden indicar que en Arslantepe, para los adobes de forma muy irregular de los edificios más antiguos, no se completaba el proceso de secado al sol, con el fin de guardar mayor plasticidad. Sin embargo, no es posible comprobar si estos hallazgos deriven de una intencionalidad en la técnica de construcción o sean vinculados a factores completamente ocasionales, como las interrupciones durante la edificación. Con respecto al relleno de la Pirámide de La Joya, la estrategia de control de las presiones internas de los rellenos por alternar bloques arcillosos y arenosos demuestra que los constructores supieron sacar ventaja de la calidad de cada clase de suelo disponible en los alrededores.

En conclusión, los datos recogidos sobre la arquitectura de tierra en éstos dos sitios diferentes muestran un diseño arquitectónico similar, con edificios rigidos con muros de adobe y techos planos de madera recubiertos por aplanados de barro. El hecho que tales construcciones son viables en zonas con regímenes de temperatura y precipitación muy distintas, semiárido templado en el caso de Arslantepe, trópico húmedo en el caso de La Joya, demuestra el valor de este tipo de arquitectura. Sin embargo, la similitud del resultado se obtuvo a partir de materias primas muy distintas: arcillas caoliníticas en uno, smectitas en otro, que crearon sus propios requisitos de manejo, al mezclarlos con minerales y fibras vegetales para controlar su estabilidad. De los cálculos estructurales para la distribución de las cargas hasta la selección del material más adecuado para cada función, de la "tecnología de los agregados" para la pasta de adobes y aplanados hasta la experimentación en la mezcla de los materiales, el secreto del éxito de la arquitectura de la tierra parece haber estado trasmitido efectivamente a través del tiempo. Se seleccionaban los materiales considerados como los más adecuados en función del material disponible y de las condiciones climáticas.

\section{Agradecimientos}

Al equipo de la Misión Arqueológica Italiana en Anatolia Oriental - MAIAO, en particular a su directora Marcella Frangipane, por autorizar el uso de los datos aquí presentados, y al topógrafo Dr. Corrado Alvaro, por su valiosa colaboración. A los especialistas que efectuaron la caracterización del material de Arslantepe: para los análisis XRF y XRD en muestras no orientadas, la Mtra. Fabiola Ferrante y el Prof. Raimondo Quaresima del Laboratorio de Química Aplicada, en el Instituto de Ingeniería de la Universidad de L'Aquila; para las medidas de densidad y porosidad, el Dr. Ing. Alexander Karamanov del Instituto de Química y Física de la Academia de Ciencias de Bulgaria; para los análisis de XRD en muestras orientadas, el Dr. Sergio Lo Mastro y el Prof. Ciriaco Giampaolo del laboratorio del Instituto de Ciencias Geológicas de la Universidad Roma 3; y para las micrografías por microscopio electrónico de barrido la Dra. María Giammatteo del Centro de Microscopia de la Universidad de L'Aquila.

A los especialistas de la UNAM que efectuaron la 
caracterización del material de La Joya: Dr. Luis Silva, del Instituto de Geología, para el estudio petrográfico; Dra. Patricia Girón, del Laboratorio de Fluorescencia de Rayos $\mathrm{X}$, Departamento de Geoquímica del Instituto de Geología, para los análisis químicos de XRD y XRF; Dr. Miguel Angel Canseco del Instituto de Investigación de Materiales, para la espectroscopía FT-IR. Al Dr. Sergey Sedov, del Instituto de Geología, quien a través de una segunda revisión de las láminas delgadas apoyó esta investigación al darle nuevos estímulos.

El proyecto arqueológico inició a finales de 2004 y tuvo el apoyo financiero de la UNAM (IIA y DGAPA, proyecto PAPIIT IN305503 otorgado a C. Navarrete y A. Daneels, PASPA 2007, PAPIIT IN405009) y las fundaciones norteamericanas FAMSI y Dumbarton Oaks. Hoy en día la investigación se enlaza con un proyecto CONACYT para la realización de estudios estructurales y composicionales de adobes, rellenos y recubrimientos de tierra obtenidos de las excavaciones, con el objetivo de reconstruir la técnica constructiva en tierra prehispánica.

\section{Referencias}

Achenza, M., Sanna, U., 2006, Il manuale tematico della terra cruda. Manuali di recupero dei centri storici della Sardegna: Cagliari, DEI, $126 \mathrm{p}$.

Alva-Balderrama, A., 2003, De la arquitectura de tierra y la conservación de lo que fue la más alta tecnología, en Feira internacional do património histórico, seminario Património, informaçao e novas tecnologías: Lisba, Direcção Geral dos Edifícios e Monumentos Nacionais, 7-13.

Alvaro, C., 2004, Il più antico complesso palatino: architettura del palazzo di Arslantepe, in Frangipane, M. (ed.), Alle origini del potere. Arslantepe collina dei leoni: Milano, Electa, 60-61.

Amici, C.M., 2008, Survey and Technical Analysis: a Must for Understanding Monuments, in Marchetti N. y Thuesen I. (eds.), ARCHAIA: Case studies onresearch, planning, characterisation, conservation and management of archaeological sites, BAR International Series 1877: Oxford, Archaeopress, 141-52.

Avrami, E., Guillaud, H., Hardy, M., 2008, Terra Literature Review, An overview of research in earthen architecture conservation: Los Angeles, The Getty Conservation Institute $156 \mathrm{p}$.

Daneels, A., 2008a, Monumental earthen architecture at La Joya, Veracruz, Mexico: Crystal River, Foundation for the Advancement of Mesoamerican Studies, reporte técnico, disponible en: $<$ http:// www.famsi.org/reports/07021> consultado el 14 de marzo 2012.

Daneels, A., 2008b, La Joya pyramid, central Veracruz, Mexico: classic period earthen architecture: Washington D.C., Dumbarton Oaks - Trustees of the Harvard University, reporte técnico, disponible en $<\mathrm{http} / / / \mathrm{www} . d o a k s . o r g /$ research/pre_columbian/doaks_pco_ project_grant_report_2007.html $>$ consultado consultado el 14 de marzo del 2012.

Daneels, A., Guerrero-Baca, L.F., 2011, Earthen architecture in the tropical lowlands of Mexico: a forgotten millenary building technique: APT Bulletin, 42 (1).

Folk, R.L., 1951, A comparison chart for visual percentage estimation: Journal of Sedimentary Research, 21, 32-33.
Frangipane, M., 2000, The Late Chalcolithic/EB I sequence at Arslantepe. Chronological and cultural remarks from a frontier site, in Marro C. y Hauptmann H. (eds.), Chronologies des pays du Caucase et de l'Euphrate aux IVe-IIIe millénaires Actes du colloque international organisé par l'Institut français d'étudesanatoliennes d'Istanbul (IFEA): Paris, Institut français d'études anatoliennes d'Istanbul, 439-471.

Frangipane, M., 2002, Non-Uruk developments and Uruk-linked features on the northern borders of Greater Mesopotamia, in Postgate, J. N. (ed.), Artefacts ofcomplexity: tracking the Uruk in the Near East Warminster Wiltshire, British School of Archaeology in Iraq, 123-48.

Galdieri, E., 1982, Le meraviglie dell'architettura in terra cruda: Bari, Laterza, $305 \mathrm{p}$.

Gandreau, D., Delboy, L. (CRAterre-ENSAG), 2010, World Heritage, Inventory and condition of properties built with earth, United Nations Educational, Scientific and Cultural Organization (UNESCO), 2007, disponible en $<$ http://whc.unesco.org/uploads/activities/documents/ activity-21-7.pdf $>$, >, consultado 13 de marzo de 2011.

Giampaolo, C., Lo Mastro, S., 2000, Analisi (semi)quantitativa delle argille mediante diffrazione a raggi X: Incontri Scientifici, 2, 109-146.

Girón, P., 2010, Protocolo de Determinación de Textura - Segregación, Anexo 14 del informe técnico parcial 2010 del proyecto PAPIIT IN 405009 "Palacios de Tierra en el Trópico Húmedo", responsable Annick J. E. Daneels. Dirección General de Asuntos del Personal Académico. Universidad Nacional Autónoma de México. México D.F.

Guerrero-Baca, L.F., 2007, Arquitectura en tierra. Hacia la recuperación de una cultura constructiva: Revista Apuntes, 20 (2), 182-201.

Guerrero-Baca, L.F., Uviña, F., 2009, La cal como estabilizante de elementos constructivos de tierra (resumen), in Adobe USA $2009-5^{\text {th }}$ InternationaConference on Earth Architectur: El Rit, New Mexico, The Adobe Association of the Southwest, 195-205.

Instituto Nacional de Estadística y Geografia e Informática (INEGI), 1992, Carta geográfica, escala 1:4000000., 1 mapa.

Liberotti, G., Quaresima, R., 2010, Building materials in the 4th and early 3rd millennium monumental architecture at Arslantepe: mudbricks and plaster, in Frangipane, M. (ed.), Economic centralization in Formative States. Thearchaeological reconstruction of the economic system the 4th millennium Arslantepe: Roma, Università La Sapienza, 73-80.

Matero, F., Del Bono, E., Fong, K.L., Johansen, R., Barrow, J., 2000, Condition and treatment history as prologue to site conservation at Casa Grande Ruins National Monument (resumen), in Terra 2000: 8th International Conference on the study and Conservation of Earthen Architecture: Torquay, Devon, United Kingdom, James and James, 52-64.

Palmieri, A.M., 1978, Studio sedimentologico dell'area nord-occidentale di Arslantepe La Ricerca Scientifica, 100, 353-364.

Palmieri, A.M., Marcolongo, B., 1983, Environment, water supply and cultural development at Arslantepe Origini, 12/2, 619-28.

Reddy, K.R., 2002, Engineering Properties Of Soils Based On Laboratory Testing: Chicago, University of Illinois, 60-73 p.

Sadori, L., Susanna, F., Balossi-Restelli, F., 2008, Collapsed beams and wooden remains from a $3200 \mathrm{BC}$ temple and palace at Arslantepe - Malatya, Turkey (resumen), in The 3rd international meeting of anthracology, 2004: Cavallino, Lecce, Italy, Archeopress, 103-116.

Manuscrito recibido: Noviembre 11, 2010.

Manuscrito corregido recibido: Septiembre 20, 2011.

Manuscrito aceptado: Enero 31, 2012. 